\title{
Unusual spin dynamics in the low-temperature magnetically ordered state of Ag3Lilr206
}

\author{
Atasi Chakraborty, Vinod Kumar, Sanjay Bachhar, N. Büttgen, K. \\ Yokoyama, P. K. Biswas, V. Siruguri, Sumiran Pujari, I. Dasgupta, \\ and A. V. Mahajan
}

\section{Published version information}

Citation: A Chakraborty et al. Unusual spin dynamics in the low-temperature magnetically ordered state of Ag3Lilr206. Phys Rev B 104, no. 11 (2021): 115106

DOI: $\underline{10.1103 / P h y s R e v B .104 .115106}$

This version is made available in accordance with publisher policies. Please cite only the published version using the reference above. This is the citation assigned by the publisher at the time of issuing the APV. Please check the publisher's website for any updates. 


\title{
Unusual spin dynamics in the low-temperature magnetically ordered state of $\operatorname{Ag}_{3} \operatorname{LiIr}_{2} \mathrm{O}_{6}$
}

\author{
Atasi Chakraborty, ${ }^{1, *}$ Vinod Kumar $\odot,{ }^{2,}{ }^{*}$ Sanjay Bachhar $\odot,{ }^{2}$ N. Büttgen, ${ }^{3}$ K. Yokoyama $\odot,{ }^{4}$ P. K. Biswas, ${ }^{4}$ V. Siruguri, ${ }^{5}$ \\ Sumiran Pujari $\odot,{ }^{2}$ I. Dasgupta, ${ }^{1}$ and A. V. Mahajan $\odot^{2, \dagger}$ \\ ${ }^{1}$ School of Physical Sciences, Indian Association for the Cultivation of Science, Jadavpur, Kolkata 700032, India \\ ${ }^{2}$ Department of Physics, Indian Institute of Technology Bombay, Powai, Mumbai 400076, India \\ ${ }^{3}$ Experimentalphysik V, Elektronische Korrelationen und Magnetismus, Institut für Physik, \\ Universität Augsburg, 86135 Augsburg, Germany \\ ${ }^{4}$ ISIS Pulsed Neutron and Muon Source, STFC Rutherford Appleton Laboratory, \\ Harwell Campus, Didcot, Oxfordshire OX110QX, United Kingdom \\ ${ }^{5}$ UGC-DAE-Consortium for Scientific Research Mumbai Centre, Bhabha Atomic Research Centre, Mumbai 400085, India
}

(Received 2 May 2021; revised 6 August 2021; accepted 10 August 2021; published 7 September 2021)

\begin{abstract}
Recently, there have been contrary claims of Kitaev spin-liquid behavior and ordered behavior in the honeycomb compound $\mathrm{Ag}_{3} \mathrm{LiIr}_{2} \mathrm{O}_{6}$ based on various experimental signatures. Our investigations on this system reveal a low-temperature ordered state with persistent dynamics down to the lowest temperatures. Magnetic order is confirmed by clear oscillations in the muon spin relaxation ( $\mu \mathrm{SR})$ time spectrum below $9 \mathrm{~K}$ until $52 \mathrm{mK}$. Coincidentally in ${ }^{7} \mathrm{Li}$ nuclear magnetic resonance, a wipeout of the signal is observed below $\sim 10 \mathrm{~K}$, which again strongly indicates magnetic order in the low-temperature regime. This is supported by our density functional theory calculations which show an appreciable Heisenberg exchange term in the spin Hamiltonian that favors magnetic ordering. The ${ }^{7} \mathrm{Li}$ shift and spin-lattice relaxation rate also show anomalies at $\sim 50 \mathrm{~K}$. They are likely related to the onset of dynamic magnetic correlations, but their origin is not completely clear. Detailed analysis of our $\mu \mathrm{SR}$ data is consistent with a coexistence of incommensurate Néel and striped environments. A significant and undiminished dynamical relaxation rate $(\sim 5 \mathrm{MHz})$ as seen in $\mu$ SR deep into the ordered phase indicates enhanced quantum fluctuations in the ordered state.
\end{abstract}

DOI: 10.1103/PhysRevB.104.115106

\section{INTRODUCTION}

Kitaev's seminal proposal of bond-dependent magnetic interactions stabilizing a novel $Z_{2}$ spin-liquid ground state with Majorana excitations, followed by the important materialspecific advance of Jackeli and Khaliullin [1] has triggered significant experimental effort to synthesize such materials. They advocated honeycomb lattice structures of $4 d / 5 d$ element-based oxides with edge-sharing oxygen octahedra and strong spin-orbit coupling as having the necessary ingredients to host the Kitaev model [2]. Several promising candidates with such layered honeycomb structure have since been investigated: $\mathrm{Na}_{2} \mathrm{IrO}_{3}$ [3-5], $\alpha-\mathrm{Li}_{2} \mathrm{IrO}_{3}$ (as also its threedimensional polymorphs) [6,7], and $\alpha-\mathrm{RuCl}_{3}[8,9]$. However, it has been revealed that these materials order magnetically [9-15] due to the presence of Heisenberg and other nonKitaev terms, and the fingerprint of the Kitaev interactions may only be realized either at higher temperatures or under application of a magnetic field.

In this family of materials a new addition has been $\mathrm{H}_{3} \mathrm{LiIr}_{2} \mathrm{O}_{6}$ [16], where all of the interlayer $\mathrm{Li}^{+}$ions of $\alpha$ $\mathrm{Li}_{2} \mathrm{IrO}_{3}$ (LIO) are replaced by $\mathrm{H}^{+}$ions, retaining the $\mathrm{LiIr}_{2} \mathrm{O}_{6}$ planes. Various measurements have confirmed the absence

\footnotetext{
*These authors contributed equally to this work.

${ }^{\dagger}$ Corresponding author: mahajan@phy.iitb.ac.in
}

of magnetic ordering down to $0.05 \mathrm{~K}$ in $\mathrm{H}_{3} \mathrm{LiIr}_{2} \mathrm{O}_{6}$ [17] which has been argued to be a spin-orbit entangled quantum spin liquid [17]. To complicate matters further, x-ray diffraction has revealed the presence of stacking faults between the honeycomb planes [16], and the low-temperature behavior was attributed to local moments induced by these defects. Theoretical ab initio calculations have also shown for these systems that although the bond-dependent Kitaev interactions are significant, the Heisenberg and other nonKitaev terms are not negligible. It has been suggested that these systems lie close to the tricritical point between ferromagnetic, zigzag, and incommensurate spiral order resulting in the absence of magnetic order [18]. Calculations further reveal that the interlayer O-H-O geometry as well as lack of hydrogen order also strongly influence the Kitaev and other exchange interactions having strong impact on its magnetic properties $[18,19]$.

Very recently, the compound $\mathrm{Ag}_{3} \mathrm{LiIr}_{2} \mathrm{O}_{6}$ (ALIO) has been synthesized [20], where proximate Kitaev spin-liquid physics has been claimed based on the scaling behavior of various thermodynamic quantities in the presence of quenched disorder and a two-step release of magnetic entropy. Replacement of the lighter $\mathrm{H}^{+}$ions by the heavier $\mathrm{Ag}^{+}$ions leads to an increase in the interlayer separation, which can significantly influence the various magnetic exchange interactions. Estimates of the magnetic interactions using $a b$ initio study are currently lacking for this system. 
We have been working on the hexagonal $\mathrm{Ag}_{3} \mathrm{Li}_{2} \mathrm{O}_{6}$ ( $M=\mathrm{Mn}, \mathrm{Ru}, \mathrm{Ir})$ system with the intention of developing a comprehensive understanding of this honeycomb system. The Mn-based ( $3 d^{3}$ or $S=3 / 2$ ) system exhibits long-range order below about $50 \mathrm{~K}$. There is also evidence of BerezinskiiKosterlitz-Thouless behavior from an analysis of the electron spin resonance line broadening data near the transition temperature [21]. The Ru-based system $\left(4 d^{4}\right.$, which might be $S=1$ or $J=0$ ) was expected to be a possible candidate for excitonic magnetism [22]. Our investigations, however, revealed that spin-orbit coupling might not be significant in this case. But the expected long-range ordered state was not seen in spite of the apparently unfrustrated geometry of the honeycomb lattice. Rather, the local moments were found to be on the borderline of being dynamic and static at low temperatures, based on muon spin relaxation $(\mu \mathrm{SR})$ data [23]. Such behavior is likely driven by higher order biquadratic or ring exchange terms in the Hamiltonian over and above the usual Heisenberg couplings.

Continuing our investigations in this series of systems, we focus in this paper on the Ir analog containing $\mathrm{Ir}^{4+}$ ions $\left(J_{\text {eff }}=1 / 2\right)$ with the purpose of looking for possible Kitaev spin-liquid physics due to the enhanced spin-orbit coupling of the Ir moments. Our experimental and theoretical results as itemized below are, however, quite far from such expectations:

(1) We observe clear oscillations in $\mu$ SR relaxation data below $\sim 9 \mathrm{~K}$ providing strong evidence for magnetic order below this temperature. Analysis of the muon data in the ordered state, complemented with density function theory (DFT) simulations of the muon stopping site points towards the coexistence of incommensurate Néel and stripe ordered magnetic domains.

(2) We also provide theoretical estimates for the various magnetic interactions in ALIO via DFT-based computations. We find the ratio of the nearest-neighbor Kitaev exchange $\left(K_{1}\right)$ to the Heisenberg exchange $\left(J_{1}\right)$ to be in the range $\left|\frac{K_{1}}{J_{1}}\right| \sim 2.0$ 3.5, placing the ALIO system far from the pure Kitaev limit, and closer to the phase boundaries between stripe, Néel, and $120^{\circ}$ order of the phase diagram [24].

(3) We observe two anomalies in the ${ }^{7} \mathrm{Li}$ nuclear magnetic resonance (NMR) shift variation with temperature. The first one that is present at $T \sim 10 \mathrm{~K}$ clearly signifies the onset of magnetic long-range order as seen from the so-called wipeout of the NMR signal, which agrees with our conclusions from muon data.

(4) The second anomaly is a broad maximum at about $50 \mathrm{~K}$ in the temperature variation of the ${ }^{7} \mathrm{Li}$ NMR line shift (which tracks the intrinsic spin susceptibility) and is reminiscent of such features in quasi-one- and quasi-twodimensional Heisenberg antiferromagnetic systems due to short-range magnetic correlations. The ${ }^{7} \mathrm{Li}$ NMR $1 / T_{1}$ also has a maximum at $T \sim 50 \mathrm{~K}$. Together with the observed progressive loss of the NMR intensity at this temperature, this could be signifying dynamic short-range magnetic correlations similar to those seen in $\mathrm{CeCu}_{2} \mathrm{Si}_{2}$ [25,26].

(5) Finally, we also find a large value of the muon relaxation rate $(\sim 5 \mathrm{MHz})$ that remains essentially flat and undiminished deep into the ordered phase, i.e., down to $52 \mathrm{mK}$, which is about $1 / 200$ th of the ordering tempera- ture of $\sim 10 \mathrm{~K}$. This is quite striking and noteworthy, rather reminiscent of spin-liquid behavior [27] in spite of the unambiguous evidence for magnetic order mentioned above (i.e., clear oscillations in $\mu \mathrm{SR}$ relaxation data). We interpret this as a signature of persistent spatiotemporal fluctuations of the Néel and stripe ordered domains, possibly driven by quantum effects given our theoretical estimates of the various magnetic exchange energy scales in ALIO.

Our results are in sharp contrast with a recent report on the same compound [28]. This report was based on the low-temperature scaling behaviors as stated before, and in particular, on an apparent two-step release of magnetic entropy suggesting ALIO may be a proximate Kitaev spin liquid. We argue that the (extracted) magnetic specific heat in the high-temperature regime is very uncertain. This is simply due to the overwhelming contribution of the lattice to the total specific heat, especially in the high-temperature region $(T \gtrsim$ $30 \mathrm{~K}$ ). Consequently, the inference of the high-temperature peak (position and magnitude) and that of a two-step entropy release is rather insecure, let alone ascribing it to Majorana excitations. We note here that an even more recent report [29] has found evidence for magnetic ordering in a cleaner batch of samples in line with our observations.

The remainder of the paper is organized as follows: we start by giving the details on the structure of ALIO and relevant technical details on measurements and theoretical methods in Sec. II. We next present our main pieces of experimental evidence that establish a low-temperature magnetic ordered state coming from $\mu \mathrm{SR}$ and NMR data in Sec. III. This is followed by a presentation of our theoretical estimates for the various magnetic exchange couplings based on DFT in Sec. IV. Section V is devoted to a detailed discussion of our observations using bulk probes (heat capacity and susceptibility) and the high-temperature anomaly in NMR vis-a-vis long-range magnetic order below $10 \mathrm{~K}$ as inferred from our observations and computation, versus the Kitaev spin-liquid scenario as in Ref. [28]. Concluding remarks are given in Sec. VI.

\section{STRUCTURE AND TECHNICAL DETAILS}

ALIO crystallizes in base centered monoclinic symmetry having space group $C 2 / \mathrm{m}$. The crystal structure is shown in Fig. 1(a). The magnetic building block consists of Ir in an octahedral environment with nearest-neighbor (NN) oxygen ligands. The $\mathrm{IrO}_{6}$ octahedra form an edge-shared honeycomb geometry in the $a-b$ plane containing Li ions at the center [see Fig. 1(b)]. The honeycomb layers in ALIO are identical to its parent compound, $\alpha$-LIO but the chemical bonds between the layers are modified. The interlayer $\mathrm{Li}$ atoms in $\alpha$-LIO are octahedrally coordinated with six oxygens in the two adjacent $\mathrm{O}_{6}$ honeycomb layers, whereas the $\mathrm{Ag}$ atoms in ALIO are linearly connected to two oxygens in neighboring layers making a $180^{\circ} \mathrm{O}-\mathrm{Ag}-\mathrm{O}$ bond angle.

Polycrystalline samples of ALIO were prepared by a two-step process as described in the Supplemental Material (SM) [30]. The resulting product was $\mathrm{Ag}_{3} \mathrm{LiIr}_{2} \mathrm{O}_{6}$ as verified by laboratory $\mathrm{x}$-ray diffraction measurements using a PANalytical X'Pert Pro diffractometer using $\mathrm{Cu} K \alpha$ radiation 

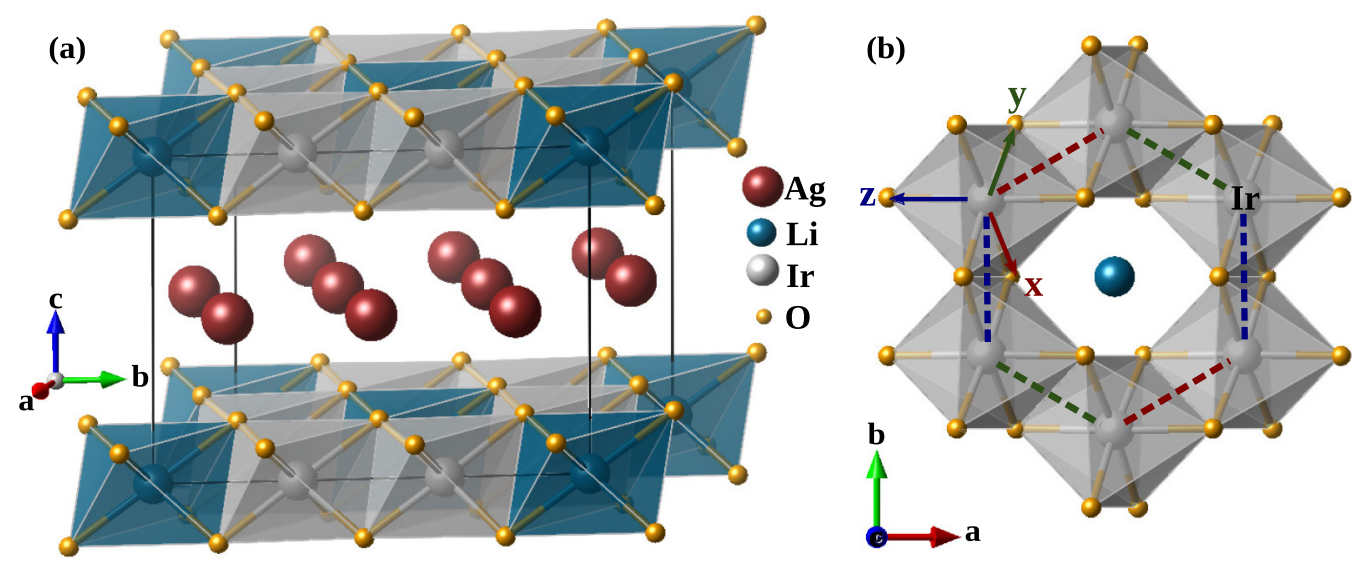

FIG. 1. (a) shows the unit cell of $\mathrm{Ag}_{3} \mathrm{LiIr}_{2} \mathrm{O}_{6}$. The edge-shared $\mathrm{IrO}_{6}$ honeycomb network is shown in (b). The $x, y, z$ local axes point toward the transition metal (Ir) to ligand $(\mathrm{O})$ direction. $X$ - (red dotted line), $Y$ - (green dotted line), and $Z$ - (blue dotted line) type Ir-Ir nearest-neighbor bonds are perpendicular to the chosen $x, y, z$ local axes, respectively.

$(\lambda=1.54182 \AA)$. Small amounts of residual $\mathrm{Ag}$ and $\alpha$ $\mathrm{Li}_{2} \mathrm{IrO}_{3}$ were detected in the x-ray diffraction pattern.

The magnetization measurements have been performed in a Quantum Design superconducting quantum interference device vibrating sample magnetometer in the temperature range $2-400 \mathrm{~K}$ and in applied fields ranging from 0 Oe to $70 \mathrm{kOe}$. The heat capacity measurements have been done in a Quantum Design physical properties measurement system in the temperature range $2-250 \mathrm{~K}$, in various field values in the range 0-90 kOe. $\mu \mathrm{SR}$ measurements were carried out using the MUSR spectrometer at the ISIS Neutron and Muon Source at the STFC Rutherford Appleton Laboratory in the UK. The powder sample was loaded on a silver sample holder to minimize the background signal. The holder was then mounted on a dilution refrigerator insert and a standard cryostat stick for measuring temperatures ranging from $50 \mathrm{mK}$ up to $150 \mathrm{~K}$. ${ }^{7} \mathrm{Li}$ NMR measurements have been performed in a fixed field of $93.954 \mathrm{kOe}$, using a Tecmag spectrometer in a continuous flow cryostat in the temperature range of 4-300 K. Measurements have also been performed in a swept field magnet down to $1.5 \mathrm{~K}$ at various frequencies (and therefore, fields). From our measurements, we obtained ${ }^{7} \mathrm{Li}$ NMR spectra, spin-lattice $\left(1 / T_{1}\right)$ and spin-spin $\left(1 / T_{2}\right)$ relaxation rates as a function of temperature in various fields.

The first-principles electronic structure calculations in the framework of DFT are carried out within the generalized gradient approximation (GGA) for the exchange-correlation functional following the Perdew-Burke-Ernzerhof prescription. We have employed the plane-wave basis as implemented within the Vienna Ab initio Simulation Package (VASP) [31,32] with projector augmented wave potentials [33,34] as well as in the $N$ th-order muffin-tin orbital (NMTO) and linear muffin-tin orbital (LMTO) basis sets as implemented in the STUTTGART code [35]. The consistency between the two sets of calculations in two choices of basis sets is cross-checked in terms of band structure, density of states, etc. The VASP calculations are done with usual values of Coulomb correlation $U$ [36] and Hund's coupling $\left(J_{H}\right)$ chosen for Ir with $U_{\text {eff }}\left(\equiv U-J_{H}\right)=$ $1.5 \mathrm{eV}$ in the Dudarev scheme [37]. The details of the VASP, LMTO, and NMTO calculations are described in the SM [30].

\section{THE LOW-TEMPERATURE ORDERED STATE OF ALIO}

\section{A. $\mu \mathrm{SR}$}

The depolarization of the muons as a function of temperature is shown in Fig. 2. At high temperatures, a slow (Gaussian-like) decay of the muon polarization is seen, whereas below about $20 \mathrm{~K}$, a faster (exponential-like) decay is discernible which gets even faster with a decrease in temperature. Finally, below about $9 \mathrm{~K}$, clear oscillations in the muon asymmetry as a function of time are seen. Fits of the time decay of the muon asymmetry at various temperatures have then been carried out to obtain the variation of the local moment dynamics with temperature. We find that at temperatures above $15 \mathrm{~K}$, the data are well fit to a product of a static KuboToyabe function with an exponential in addition to a constant background $A_{0}$, i.e., $A(t)=A_{2} G_{K T}(\Delta, t) e^{-\lambda_{2}(T) t}+A_{0}$. Here, $G_{K T}(\Delta, t)$ is the Kubo-Toyabe function which models the relaxation of muons in a Gaussian distribution of magnetic fields from nuclear moments. From these fits we obtain the

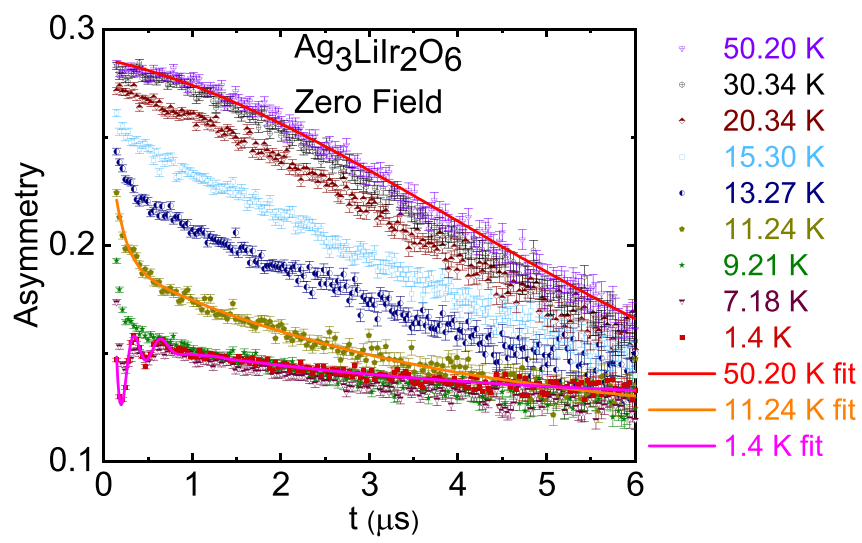

FIG. 2. Variation of the muon asymmetry with time is shown at selected temperatures. Clear oscillations are seen below about $9 \mathrm{~K}$ indicative of long-range magnetic order. Fits at some representative temperatures are shown as explained in the text. 


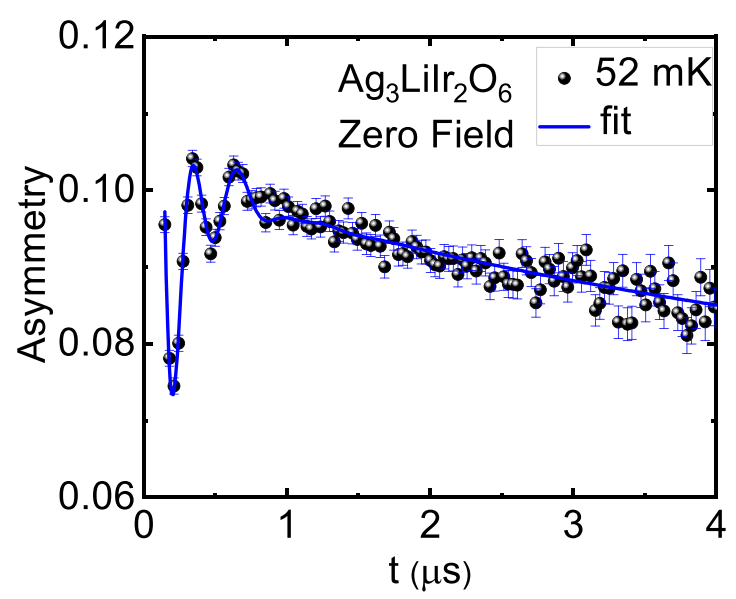

FIG. 3. Variation of the muon asymmetry with time is shown at $52 \mathrm{mK}$ together with a fit to Eq. (1). The fit parameters are nearly unchanged up to $7 \mathrm{~K}$.

field distribution $\Delta$ to be about $1.6 \mathrm{Oe}$. This value is typical of nuclear dipolar fields at the muon site, in the present case arising from ${ }^{107,109} \mathrm{Ag},{ }^{6,7} \mathrm{Li}$, and ${ }^{191,193} \mathrm{Ir}$ nuclei. The exponential term $e^{-\lambda_{2}(T) t}$ arises from the relaxation due to fluctuations of the electronic local moments. This relaxation rate $\lambda_{2}(T)$ is small at high temperatures and gradually increases as the local moment fluctuation rate gets smaller (see Fig. 4). We notice a sharper increase of $\lambda_{2}(T)$ below about $20 \mathrm{~K}$.

The intermediate region of 13 to $9 \mathrm{~K}$ shows a sharply falling muon asymmetry with $\lambda_{2}(T)$ showing a sharp increase with decreasing temperature as an approach to long-range order. Going further down in temperature, we find that below $9 \mathrm{~K}$, there are clear oscillations in the muon asymmetry as a function of time. This is a classic signature of the presence of long-range magnetic order. The temporal decay of the muon asymmetry is nearly unchanged from $7 \mathrm{~K}$ down to $52 \mathrm{mK}$. The data in the range $52 \mathrm{mK}-7 \mathrm{~K}$ were well fit (see Fig. 3) by the following equation:

$$
\begin{aligned}
A(t)= & A_{0}+A_{1} e^{-\lambda_{1}(T) t} J_{0}\left(\gamma H_{1} t\right) \\
& +A_{2} e^{-\lambda_{2}(T) t} J_{0}\left(\gamma H_{2} t\right)+A_{3} e^{-\lambda_{3}(T) t},
\end{aligned}
$$

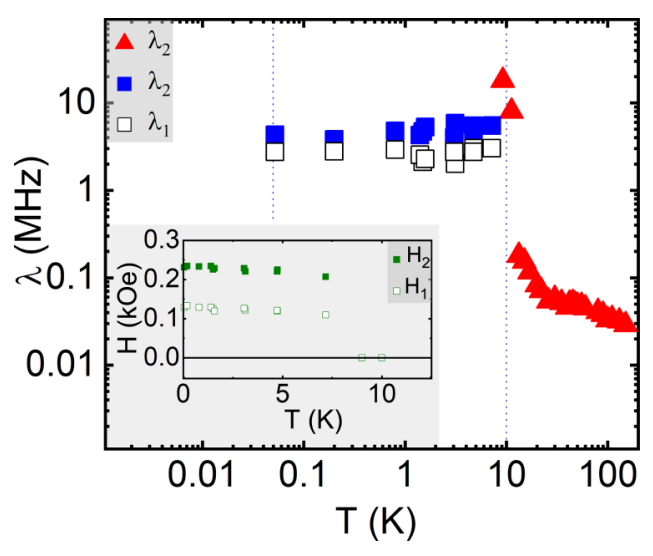

FIG. 4. Variation of the muon relaxation rate (main figure) and the local field at the muon site (inset) as a function of temperature for ALIO is shown. where $\gamma$ is the muon gyromagnetic ratio $(\gamma=2 \pi \times 135.539$ $\mathrm{MHz} /$ Tesla). The $A_{3} e^{-\lambda_{3}(T) t}$ term is ascribed to muons which are initially parallel to the internal field components and hence do not precess. As for the other significant terms, $J_{0}\left(\gamma H_{1} t\right)$ and $J_{0}\left(\gamma H_{2} t\right)$ are zeroth-order Bessel functions. In case of ordering that is commensurate with the lattice, one expects an exponentially damped sinusoidal variation of $A(t)$ in the ordered state. The Bessel function variation observed here is indicative of magnetic order incommensurate with the lattice [38] (such as for a spin density wave), where the muon experiences fields up to a maximum of $H_{1}$ or $H_{2}$, in the present case. We found that the fit was better with two Bessel functions rather than one (see SM [30] for a comparison), suggesting the presence of two types of magnetic environments for the muons. These could arise either from the presence of two kinds of regions with different spin order, or possibly from crystallographically inequivalent muon stopping sites.

From our DFT calculations (see Sec. IV for details), we conclude that while the Néel-type order has the lowest energy, the stripy phase is not much higher in energy, which lends credence to the first possibility. Thus, assuming this scenario of a single muon stopping site, the site was determined from calculations of the electrostatic energy and was found to be about $1 \AA$ from the oxygen ion (see SM [30] for details). This is similar to that found in cuprates and other oxide materials [39]. We then calculated the dipolar magnetic fields at the muon stopping site in Néel and stripy environments, respectively. The calculated field values of 139 Oe for the Néel phase and 266 Oe for the stripy phase (assuming a moment of $0.5 \mu_{B} / \mathrm{Ir}$ which is typical for $\mathrm{Ir}^{4+}$ ) are in reasonable agreement with the values of 129 and 232 Oe obtained as the averages of fit parameters $H_{1}$ and $H_{2}$, respectively, between $52 \mathrm{mK}$ and $1.4 \mathrm{~K}$ (see inset of Fig. 4).

Finally, we look at the variation of the muon relaxation rates $\lambda_{1}$ and $\lambda_{2}$ vs $T$. It shows a peak at about $9 \mathrm{~K}$, but does not fall to low values even at $52 \mathrm{mK}$ as is expected to happen for progressively slower dynamics as we go deeper into the ordered state (Fig. 4). It rather stays almost flat and undiminished at a value of about $5 \mathrm{MHz}$ in the low-temperature side, as seen in Fig. 4. We speculate that persistent spatiotemporal fluctuations of the stripy and Néel regions are responsible for this. In such a scenario, this would present an interesting example where these fluctuations persist even at temperatures more than two orders of magnitude lower than the transition temperature $(\sim 9 \mathrm{~K})$ until at least a thermal energy scale of $\sim 50 \mathrm{mK}(4.3 \mu \mathrm{eV})$. Could these then be quantum mechanical in origin?

From a quantitative point of view, the rather large value of $5 \mathrm{MHz}$ for the muon depolarization rate is quite remarkable, comparable to (or even larger than) those seen in a variety of spin liquids (e.g., see the review, Ref. [27], and the references therein; also see recent Refs. [40,41] not covered in this review). Even in $\alpha-\mathrm{RuCl}_{3}$, which has been established to be magnetically ordered, the muon relaxation rate is appreciable $(\sim 0.5 \mathrm{MHz}$ in a single crystal [42] and $\sim 2 \mathrm{MHz} / \sim 4 \mathrm{MHz}$ in a polycrystalline sample [43]) though smaller compared to ALIO. However, these observations on $\alpha-\mathrm{RuCl}_{3}$ are limited, respectively, to roughly $1 / 2$ the ordering temperature in the single crystal work [42], and about $1 / 5$ th the ordering temperature in the polycrystalline sample study [43], whereas our 


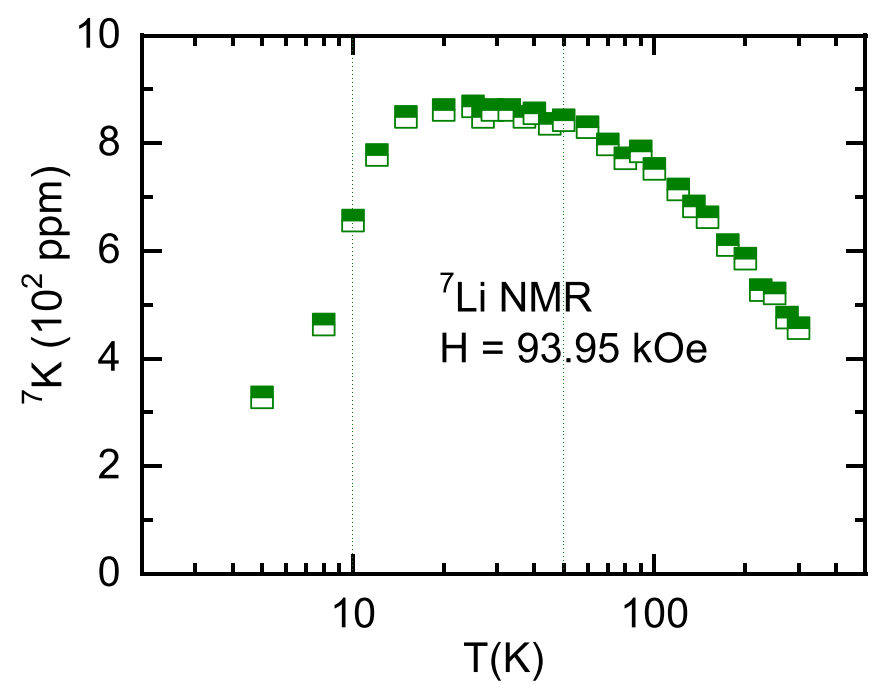

FIG. 5. The ${ }^{7} \mathrm{Li}$ NMR shift increases with decreasing temperature and then shows a broad plateau below $50 \mathrm{~K}$.

observations on ALIO go down to $1 / 200$ th of the ordering temperature and can be considered well representative of the ground state physics. This suggests that the persistent dynamics are really a feature of the magnetically ordered many-body ground state.

\section{B. NMR}

Having established the presence of magnetic order in ALIO from our zero-field $\mu$ SR data at low temperatures, we now move over to the local probe technique of NMR to examine the variation of the intrinsic spin susceptibility in the paramagnetic state, as well as to look for complementary evidence of ordering. The bulk susceptibility, on the other hand, can have a low- $T$ upturn arising from extrinsic contributions or orphan spins which may not be reflective of the intrinsic properties. We have therefore performed ${ }^{7} \mathrm{Li}$ local probe NMR measurements to determine the shift of the ${ }^{7} \mathrm{Li}$ resonance $\left({ }^{7} \mathrm{~K}\right)$ with respect to a diamagnetic reference as a function of temperature. The results are shown in Fig. 5 where it is seen that the intrinsic susceptibility (in the form of a ${ }^{7} \mathrm{~K}$ line shift) increases with decreasing temperature and then exhibits a broad plateau region below about $50 \mathrm{~K}$.

Furthermore, as NMR is a good probe of low-energy excitations, we have performed ${ }^{7} \mathrm{Li} \mathrm{NMR}$ spin-lattice relaxation rate measurements as a function of temperature. The recovery of the longitudinal nuclear magnetization after a saturating pulse sequence was well fitted with a single exponential. As shown in Fig. 6, we find that $1 / T_{1}$ increases with decreasing temperature and has a peak around $50 \mathrm{~K}$ similar to systems which show ordering. The ${ }^{7} \mathrm{~K}$ and $1 / T_{1}$ results are found independent of the applied field between 18 and $94 \mathrm{kOe}$.

We now contrast our observations with those reported in the very recent reference [44] performed on two different batches of samples (A and B) of ALIO. Wang et al. found that the cleaner sample A showed a single peak in the NMR spectrum as opposed to two peaks for sample B. The longitudinal nuclear magnetization recovery for sample A was found to be single exponential in contrast to a stretched exponential

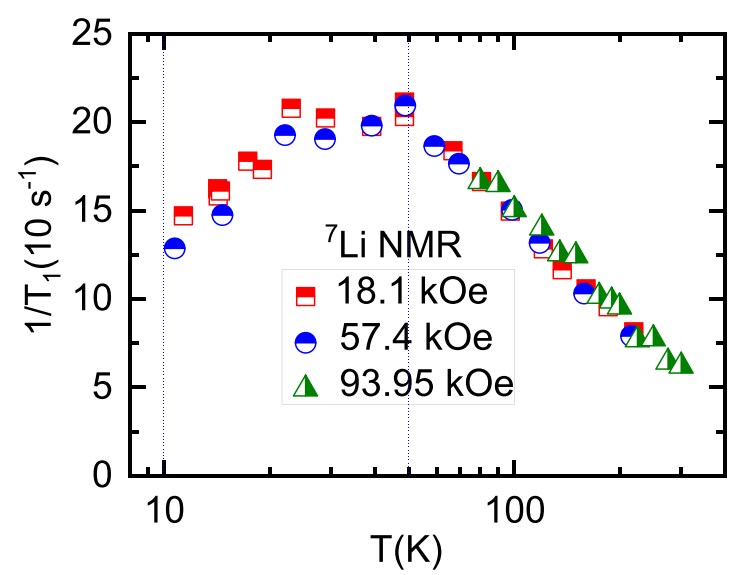

FIG. 6. The variation of he ${ }^{7} \mathrm{Li}$ NMR spin-lattice relaxation rate is shown with $T$. This also shows a peak at about $50 \mathrm{~K}$ where there is a susceptibility anomaly.

variation for sample B. Also, the values of the relaxation rate were higher for sample A compared to sample B. The near absence of a second peak in the NMR line shape of our sample of ALIO, together with a single exponential recovery in $T_{1}$ and with the absolute value of the ${ }^{7} \mathrm{Li}$ NMR relaxation rate $1 / T_{1}$ on the higher side, we conclude that our sample is similar to sample A of Ref. [44].

We also monitored the total NMR spectral intensity as a function of temperature which naturally involves the measurement of the spin-spin relaxation rate $\left(1 / T_{2}\right)$. The total spectral intensity should normally vary as a Curie law due to a Curie variation of the nuclear magnetization. Hence, the product of the nuclear magnetization $M_{0}$ and $T$ should remain constant with temperature in case the same number of nuclei contribute to the signal at all temperatures. Our results for $1 / T_{2}$ and $M_{0} T$ are shown in Fig. 7. We observe the onset of a decrease in intensity already around $50 \mathrm{~K}$ and a near complete wipeout below $10 \mathrm{~K}$. The observed wipeout is a classic signature of the onset of long-range magnetic ordering. An anomaly is also seen in the $1 / T_{2}$ data around $10 \mathrm{~K}$. We thus conclude that there is an onset of short-range correlations around $50 \mathrm{~K}$

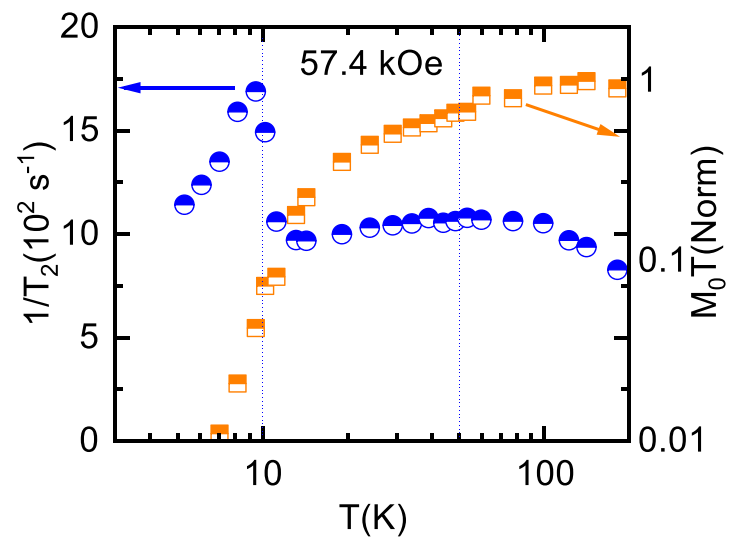

FIG. 7. The variation of the ${ }^{7} \mathrm{Li}$ NMR spin-spin relaxation rate (left $y$ axis) is shown with $T$. The right $y$ axis shows the product of the nuclear magnetization and temperature normalized to the maximum, as a function of $T$. 
TABLE I. Bond lengths are given in $\AA$ and bond angles are in degrees $\left({ }^{\circ}\right)$.

\begin{tabular}{lllll}
\hline \hline & $\begin{array}{l}\alpha-\mathrm{Li}_{2} \mathrm{IrO}_{3} \\
\text { Parameters }\end{array}$ & $\begin{array}{l}\mathrm{H}_{3} \mathrm{LiIr}_{2} \mathrm{O}_{6} \\
\text { (expt.) [45] }\end{array}$ & $\begin{array}{c}\mathrm{Ag}_{3} \mathrm{LiIr}_{2} \mathrm{O}_{6} \\
\text { (expt.) [20] }\end{array}$ & $\begin{array}{c}\mathrm{Ag}_{3} \mathrm{LiIr}_{2} \mathrm{O}_{6} \\
\text { (relax) }\end{array}$ \\
\hline $\begin{array}{l}\text { Ir-Ir distance } \\
(X / Y \text { bond) }\end{array}$ & 2.98 & 3.10 & 3.06 & 3.04 \\
$\begin{array}{l}\text { Ir-Ir distance } \\
(Z \text { bond) }\end{array}$ & 2.98 & 3.05 & 3.04 & 3.08 \\
$\begin{array}{l}\text { Ir-O-Ir angle } \\
(X / Y \text { bond) }\end{array}$ & 94.74 & 99.77 & 98.55 & 97.76 \\
$\begin{array}{l}\text { Ir-O-Ir angle } \\
(Z \text { bond) }\end{array}$ & 95.42 & 99.03 & 92.43 & 100.08 \\
$\begin{array}{l}\text { (Li,H,Ag)-O } \\
\text { distance }\end{array}$ & $1.88,2.13$ & $1.23,1.27$ & $1.94,2.09$ & $2.08,2.10$ \\
\hline \hline
\end{tabular}

and eventually long-range magnetic ordering sets in at about $10 \mathrm{~K}$.

\section{FIRST-PRINCIPLES ELECTRONIC STRUCTURE CALCULATIONS}

In order to gain a microscopic understanding of the electronic and magnetic behavior, we first optimized the crystal structure parameters using first-principles density functional theory calculations. The crystal structure of ALIO has been shown in Fig. 1, and we recall that the various exchange parameters, in particular the Kitaev exchange interaction, are strongly dependent on the NN bond length and bond angles. To cross-check the experimental refinement of the position of the light atoms (such as $\mathrm{O}$ ) based on x-ray diffraction, we have independently determined the structural parameters by carrying out an ionic relaxation simulation for ALIO while maintaining the crystal symmetries of space group $C 2 / m$ (see SM [30] for details). A comparison of the structural data for the experimental and the relaxed structures are presented in Table I along with the structural data of $\mathrm{H}_{3} \mathrm{LiIr}_{2} \mathrm{O}_{6}$ (HLIO) and the parent compound $\alpha$-LIO with similar stoichiometries. We find for the relaxed structure of ALIO, the Ag-O bond lengths along the O-Ag-O angle are nearly equal and substantially larger in comparison to its $\mathrm{H}$ counterpart while the Ir-O-Ir angle for the $\mathrm{Z}$ bond is nearly identical for both the systems. All the subsequent calculations are performed with this relaxed structure of ALIO.

We begin with an investigation of the electronic structure of ALIO without magnetic order. The results of our calculations are summarized in the top panel of Fig. 8, where we have plotted the total as well as the Ir projected density of states (DOS). We find that the octahedral environment of Ir splits its $d$ states into $t_{2 \mathrm{~g}}$ and $e_{\mathrm{g}}$ states by a large crystal field splitting $\left(\Delta_{C F} \sim 4.3 \mathrm{eV}\right)$ characteristic of iridates. The $t_{2 \mathrm{~g}}$ states are further split due to monoclinic distortion and host the Fermi level. The oxygen- $p$ and Ag- $d$ states are completely occupied, while the Ir- $e_{\mathrm{g}}$ and Li- $s$ states are completely empty consistent with the nominal ionic formula $\mathrm{Ag}_{3}^{1+} \mathrm{Li}^{1+} \mathrm{Ir}_{2}^{4+} \mathrm{O}_{6}^{2-}$.

In view of the large $\Delta_{C F}$, the entire physics of these systems is essentially governed by the $t_{2 \mathrm{~g}}$ states. We have therefore constructed a low-energy tight-binding model retaining only the $t_{2 \mathrm{~g}}$ states in a local basis (where the local

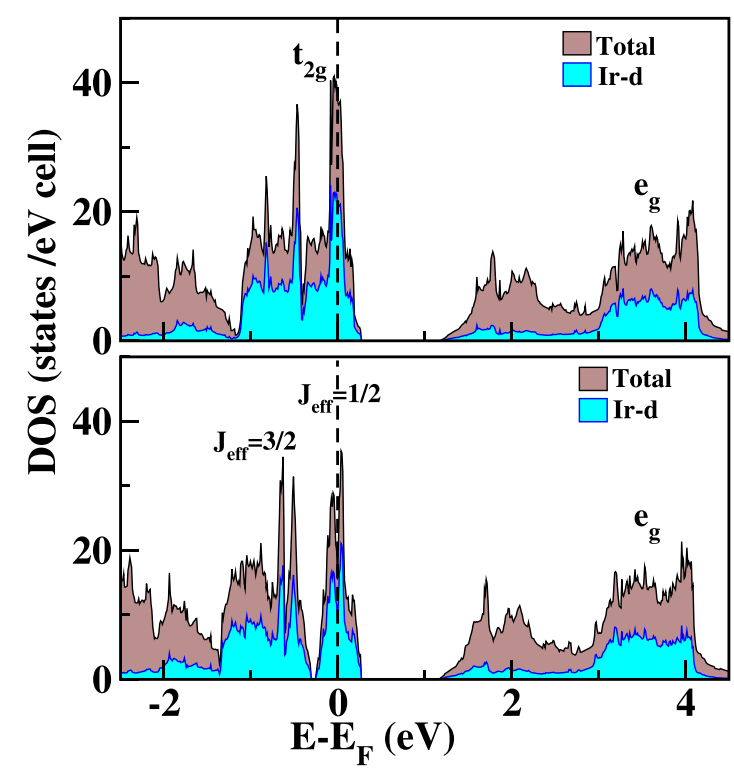

FIG. 8. Nonmagnetic total density of states (DOS) (brown shaded region) and partial Ir- $d$ DOS (cyan shaded region) are shown. Top and bottom panels show the DOS for GGA calculations without and with spin-orbit coupling.

$x, y$, and $z$ axes point towards the ligands) and downfolded all other higher degrees of freedom using the NMTO downfolding method [35]. The $C 2 / m$ group splits the $t_{2 \mathrm{~g}}$ states retaining twofold symmetry at each metal site. The various hopping interactions between the Ir atoms reveal that the hopping corresponding to the first $\mathrm{NN}$ is stronger compared to the other interactions. The $C 2 / \mathrm{m}$ space group provides two types of symmetry inequivalent nearest-neighbor bonds: (i) $Z$ bonds, parallel to the crystallographic $b$ axis, are of local $C 2 h$ symmetry; (ii) $X$ or $Y$ bonds with lower symmetry. The nearest neighbor $d$ - $d$ hopping integrals for the $Z$ bond expected for the ideal structure are extracted from NMTO downfolding calculations by suitable averaging and the values are $t_{1} \equiv t_{x z, x z}=t_{y z, y z}=-49.1 \mathrm{meV}, t_{2} \equiv t_{x z, y z}=t_{y z, x z}=$ $204.0 \mathrm{meV}, t_{3} \equiv t_{x y, x y}=-1.4 \mathrm{meV}$, and $t_{4} \equiv t_{x z, x y}=t_{x y, x z}=$ $t_{y z, x y}=t_{x y, y z}=46.2 \mathrm{meV}$. While $t_{2}$ is strongest as expected, $t_{3}$ is, however, strongly suppressed. This will have a profound impact on the exchange interactions to be discussed later in the section. We now present the effect of spin-orbit coupling (SOC) on the electronic structure of ALIO. The total and the Ir projected DOS are displayed in Fig. 8. We find that the SOC further splits the $t_{2 \mathrm{~g}}$ states into low-lying, fourfold degenerate $J_{\text {eff }}=3 / 2$ and high-lying twofold degenerate $J_{\text {eff }}=$ $1 / 2$ states. The five electrons of nominal $\mathrm{Ir}^{4+}$ completely fill the $J_{\text {eff }}=3 / 2$ states, while the $J_{\text {eff }}=1 / 2$ state is half-filled, which upon inclusion of a moderate Hubbard interaction $U$ makes the system insulating. In the limit $U \gg t$, the holes occupying the $J_{\text {eff }}=1 / 2$ states are nearly localized on the metal sites and the low-energy degrees of freedom are pseudospin-1/2 variables $\mathbf{S}_{\mathbf{i}}$ connected to the $J_{\text {eff }}=1 / 2$ states.

This spin-orbit entangled pseudospin state of Ir atom on a honeycomb lattice in the strong coupling limit hosts bond-dependent anisotropic Kitaev exchanges in addition to the usual isotropic Heisenberg exchange terms. The 
nearest-neighbor spin Hamiltonian may be written as $H_{\text {spin }}=$ $\sum_{\langle i, j\rangle} \mathbf{S}_{i} \cdot \mathbf{J}_{i j} \cdot \mathbf{S}_{j}$, where $\mathbf{J}_{i j}$ is a $3 \times 3$ symmetric matrix due to the presence of local inversion symmetry and is given by

$$
\left[\begin{array}{ccc}
J_{1} & \Gamma_{1} & \Gamma_{1}^{\prime} \\
\Gamma_{1} & J_{1} & \Gamma_{1}^{\prime} \\
\Gamma_{1}^{\prime} & \Gamma_{1}^{\prime} & J_{1}+K_{1}
\end{array}\right] .
$$

The various parameters of the spin Hamiltonian [Eq. (2)] are calculated following Refs. [15,24] using the hoppings obtained from the NMTO downfolding method mentioned earlier and neglecting the crystal field terms. For $U=1.7 \mathrm{eV}$, $J_{H}=0.3 \mathrm{eV}$, and $\lambda=0.4 \mathrm{eV}$ suitable for ALIO [15], we obtain for the spin Hamiltonian $\left(J_{1}, K_{1}, \Gamma_{1}, \Gamma_{1}^{\prime}\right)$ in Eq. (2) as $(+3.19,-11.4,-1.3,-2.99) \mathrm{meV}$ and $(2.86,-5.85,-0.67$, $-1.54) \mathrm{meV}$ by using the strong coupling expressions from Ref. [15] and Ref. [24], respectively. The reported values of $\left(J_{1}, K_{1}, \Gamma_{1}, \Gamma_{1}^{\prime}\right)$ from two different studies on a similarly stoichiometric HLIO structure that has been suggested to be a Kitaev quantum spin liquid [17] are $(1.8 \mathrm{meV},-12.0 \mathrm{meV}$, $-0.2 \mathrm{meV},-3.2 \mathrm{meV})$ [19] and $(-1.3 \mathrm{meV},-15.4 \mathrm{meV}$, $+1.5 \mathrm{meV},-5.1 \mathrm{meV}$ ) [18], respectively. While the ferromagnetic nature of $K_{\mathrm{z}}$ obtained for ALIO is similar to that of HLIO, the magnitude of $\left|\frac{K_{1}}{J_{1}}\right|$ is $2-3.5$. This is much smaller in comparison to HLIO $(8.5-12.5)$. It is therefore likelier that ALIO will order like the parent compound $\alpha$-LIO [7], for which the (two) reported estimates of $\left(J_{1}, K_{1}, \Gamma_{1}, \Gamma_{1}^{\prime}\right)$ are $(-4.6 \mathrm{meV},-4.2 \mathrm{meV},+11.6 \mathrm{meV},-4.3 \mathrm{meV})$ and $(-3.1 \mathrm{meV},-6.3 \mathrm{meV},+9.4 \mathrm{meV},-0.1 \mathrm{meV})$ [15].

Perturbative analysis [24] estimates that $J \sim \frac{t_{d d}^{2}}{U}$ and $K \sim$ $\frac{t_{p d}^{4}}{\Delta_{p d}^{2}} \frac{J_{H}}{U^{2}}$ with the Heisenberg term predominantly governed by direct exchange, while the Kitaev interaction is mostly due to superexchange processes along the Ir-O-Ir paths. Here, $t_{d d}$ and $t_{p d}$ stand for the hopping amplitudes between $d$ orbitals of neighboring Ir ions and between Ir- $d$ and O- $p$ states, respectively. $\Delta_{p d}$ is the charge-transfer energy. Unlike Li-s in honeycomb $\alpha$-LIO, the Ag- $d$ orbitals form strong covalent bonds with ligand O- $p$ orbitals [seen from the crystal orbital Hamiltonian population plot in SM [30]] resulting in strong $d$ $p$ mixing. This modifies the Ir-O-Ir superexchange interaction and enhances the Kitaev term $(K)$. In comparison to $\alpha$-LIO $\left(\Gamma_{1}=11.6 \mathrm{meV}\right)$ [15], the increased Ir-O-Ir bond angle in ALIO suppresses $t_{3}$ and leads to the reduced $\Gamma_{1}$ similar to HLIO. We have also calculated the second and third neighbor interaction strengths and these are found to be much weaker, $\left(J_{2 \mathrm{nd}}<6 \%\right.$ of $J_{\mathrm{NN}} ; J_{3 \mathrm{rd}}<1 \%$ of $\left.J_{\mathrm{NN}}\right)$. Our calculated parameters place ALIO in the vicinity of the striped phase and the Néel antiferromagnetic (AFM) phase in the phase diagram reported in Ref. [24]. The $120^{\circ}$ magnetic phase is, as well, in close proximity for these parameters. To determine the likely ground state of ALIO, we have considered several magnetic configurations whose spin and orbital moments are consistent with the $J_{\text {eff }}=1 / 2$ state of $\mathrm{Ir}$, and calculated their energies within the GGA $+\mathrm{SOC}+U$ scheme (see Fig. 9). Apart from the zigzag AFM order that has been observed in the parent compound $\alpha$-LIO [7], we have also examined three other representative magnetic orders: ferromagnet (FM), Néel AFM, and stripe AFM, which have been observed in honeycomb materials for the $q=0$ magnetic structure [4,9]. Whereas the

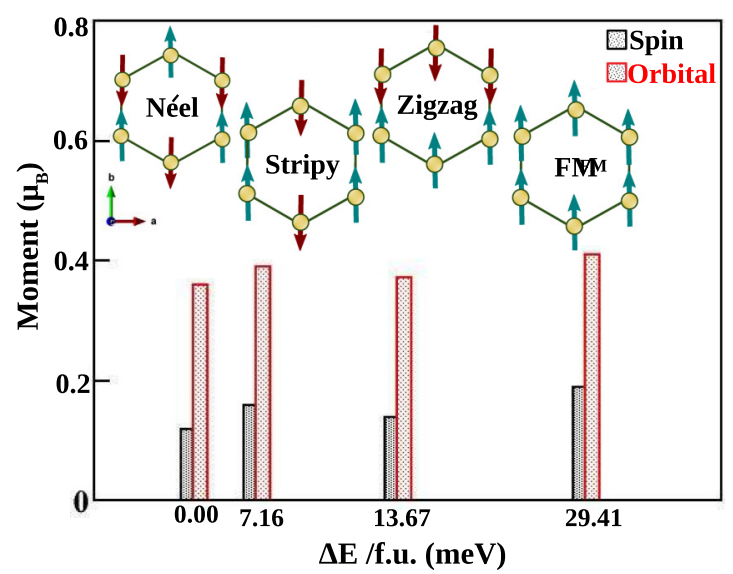

FIG. 9. Comparison of different magnetic configurations within $\mathrm{GGA}+\mathrm{SOC}+U$ calculation

Néel AFM order has the lowest energy among the chosen configurations, the stripy phase is not much higher in energy. The $q \neq 0$ solutions and the incommensurate phases are not taken into consideration, which may also dictate the ground state magnetism, especially the $120^{\circ}$ ordered phase.

\section{DISCUSSION ON BULK PROBES AND HIGH-TEMPERATURE ANOMALY}

In this section, we discuss bulk probes-heat capacity (Fig. 10) and magnetic susceptibility (Fig. 11) - and the high-temperature anomaly in the NMR data in light of the low-temperature ordered state established in the previous sections. We start with heat capacity data. As is well known, the measured heat capacity contains a contribution from the crystal lattice degrees of freedom in addition to magnetic contributions. At higher temperatures, it is the lattice contribution which generally dominates the heat capacity. To determine the lattice part, one can make use of structurally analogous nonmagnetic variants of the given compound if available. In such cases, corrections are necessary in inferring the lattice contribution of the magnetic compound from that

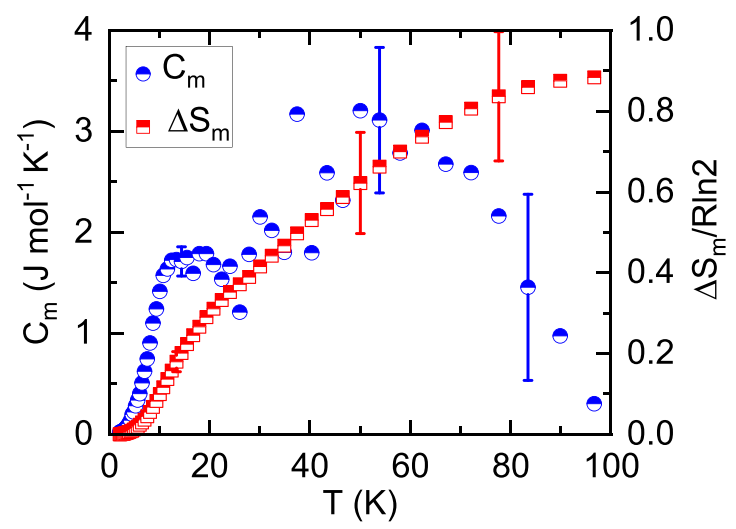

FIG. 10 . The magnetic heat capacity $\left(C_{m}\right)$ and the entropy change $\left(S_{m}\right)$ are plotted in units of $R \ln 2$ as a function of temperature. Representative error bars are given at a few temperatures. Anomalies are observed at $T_{H} \simeq 50 \mathrm{~K}$ and $T_{L} \simeq 13 \mathrm{~K}$. 


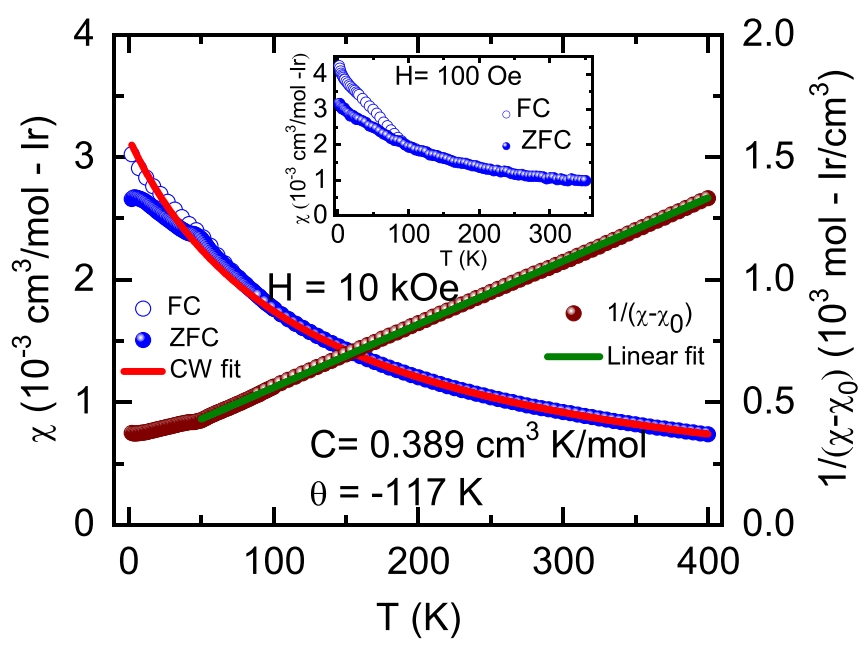

FIG. 11. Variation of $\chi$ with temperature is shown together with the inverse susceptibility. The inset shows that the susceptibility measured in a field of 100 Oe has a clear bifurcation below $100 \mathrm{~K}$ suggesting static moment formation.

of the nonmagnetic analog to account for the differences in their effective Debye temperatures of the two compounds due to the different ionic masses and unit cell volumes. Following such a correction, the heat capacities of the two compounds must coincide in a high-temperature region where the magnetic contribution is negligible. In Refs. [28,29], the authors have made use of $\mathrm{Ag}_{3} \mathrm{LiSn}_{2} \mathrm{O}_{6}$ (ALSO) as the nonmagnetic analog. However, it appears that no correction has been applied before subtracting these data from the measured heat capacity of ALIO. This can be seen in Fig. 3(a) of Ref. [28] and Fig. 2(a) of Ref. [29] where the data for ALIO and ALSO do not appear to overlap at high temperatures.

The magnetic specific heat of our sample, using the data for the structurally analogous nonmagnetic $\mathrm{Ag}_{3} \mathrm{LiTi}_{2} \mathrm{O}_{6}$ for the lattice contribution (see SM [30] for details), is given in Fig. 10. Anomalies are seen in our data at about 13 and $50 \mathrm{~K}$. Based on our NMR and $\mu$ SR data described in Sec. III, the lower-temperature anomaly is from long-range order. The higher-temperature $50 \mathrm{~K}$ anomaly could be from short-range correlations (recall the broad plateau seen in the ${ }^{7} \mathrm{Li}$ NMR shift with temperature, Fig. 5). However, it should be noted that at high temperatures, the magnetic heat capacity is quite small compared to the lattice contribution, and hence a small error in the lattice heat capacity gives rise to a large error in the magnetic heat capacity. For instance, at about $50 \mathrm{~K}$, the inferred magnetic specific heat is only about $13 \%$ of the total specific heat. Therefore, a $5 \%$ error in the lattice heat capacity will result in nearly $50 \%$ error in magnetic heat capacity at $50 \mathrm{~K}$. Consequently, the magnetic entropy change as well suffers from high uncertainty, especially in the high-temperature region. As a result, the inference of a two-stage entropy release is suspect in our opinion, which calls into question an interpretation in terms of localized and itinerant Majorana excitations. The DC magnetic susceptibility $\chi(T)=M / H$ of $\mathrm{Ag}_{3} \mathrm{LiIr}_{2} \mathrm{O}_{6}$ is shown in Fig. 11. It varies in a Curie-Weiss manner at high temperature but has an anomaly at about $50 \mathrm{~K}$. In particular, as shown in the inset of Fig. 11, in a low field of $100 \mathrm{Oe}$, there is a clear bifurcation between the zero-field-cooled (ZFC) and field-cooled (FC) curves a little below $100 \mathrm{~K}$. Such a measurement is currently not available in the literature for comparison.

Given the above bulk probe data, we revisit the hightemperature anomaly seen in NMR data which one might want to optimistically interpret as having to do with Kitaev physics. Broad anomalies, such as the one in the ${ }^{7} \mathrm{Li}$ NMR line shift of ALIO at about $50 \mathrm{~K}$ (Fig. 5), are expected in low-dimensional quantum magnets with antiferromagnetic couplings at temperatures on the scale of the dominant exchange coupling. In such a case, an anomaly is also expected in the temperature dependence of $1 / T_{1}$. It should be noted that ${ }^{7} \mathrm{Li}$ nuclei are located symmetrically with respect to the Ir moments on the lattice, which will filter out Néel-type antiferromagnetic fluctuations. However, given the evidence from $\mu \mathrm{SR}$ in Sec. III A of the eventual incommensuration below $9 \mathrm{~K}$, as also the possibility of the (incommensurate) stripy phase as a competing phase for which the fluctuations are not canceled at the ${ }^{7} \mathrm{Li}$ site, one expects now incomplete filtering at the ${ }^{7} \mathrm{Li}$ site. The appearance of a peak at $50 \mathrm{~K}$ (Fig. 6) then likely reflects a build-up of short-range dynamic magnetic correlations at the dominant exchange scale as concluded in Sec. III B. This $50-\mathrm{K}$ scale is indeed in agreement with our DFT estimates (Sec. IV) for the various exchange couplings (also, see SM [30]). On the other hand, as discussed in the NMR section (Sec. III B), the absence of a sharp divergence in $1 / T_{1}$ at the ordering temperature $(\sim 10 \mathrm{~K})$ is likely due to a wipeout effect where a large fraction of the ${ }^{7} \mathrm{Li}$ nuclei (nearly $90 \%$ as seen from Fig. 7) are already out of the window of observation by $10 \mathrm{~K}$

We now return to the bifurcation seen in the low-field ZFC/FC susceptibility data at about $100 \mathrm{~K}$ suggesting a freezing of magnetic moments (inset of Fig. 11). In higher fields, this anomaly moves to about $50 \mathrm{~K}$. If this arose from intrinsic regions, it would result in a sharp loss of the NMR spectral intensity at this temperature. On the other hand, the observed loss of NMR intensity is rather gradual as the temperature decreases from $100 \mathrm{~K}$ (Fig. 7). Further, one should have seen a reflection of the freezing of moments in the $\mu$ SR relaxation rate $\lambda_{2}$ which is a zero-field measurement. However, $\lambda_{2}$ shows a rather gentle variation with temperature with a sharper increase only below $20 \mathrm{~K}$ (Fig. 4). This then suggests that the $\mathrm{ZFC} / \mathrm{FC}$ bifurcation is extrinsic in origin, possibly related to moments localized at stacking faults that naturally occur in these systems, and one has to look for a different cause for the decrease in the NMR intensity.

We note that such a decrease of the NMR intensity has been seen in the heavy-fermion superconductor $\mathrm{CeCu}_{2} \mathrm{Ge}_{2}$ above the superconducting transition temperature as well as in its nonsuperconducting variants [25,26]. In these cases, it was ascribed to dynamical magnetic correlations arising from the itinerant physics of the "heavy-fermion band magnetism." In the present case, our DFT estimates of the Heisenberg and Kitaev terms tell us that they are comparable $\left(\left|\frac{K_{1}}{J_{1}}\right|\right.$ is about 2-3.5) and in the range of the energy scale $\sim 50 \mathrm{~K}$. As discussed earlier in this section, these competing interactions can be driving the development of short-range dynamical magnetic correlations in ALIO. Whether there is some form of itinerancy - by which, we have in mind some form of a 
quasiparticle excitation continuum [46] - in the ALIO system driven by the Kitaev terms leading to the above decrease of the NMR intensity is an open question.

\section{CONCLUSIONS}

In summary, ALIO is shown to exhibit magnetic longrange order below $9 \mathrm{~K}$. This observation is consistent with our DFT calculations which find comparable Heisenberg and Kitaev exchange couplings. Though $5 d \mathrm{Ir}^{4+}$ ions possess strong spin-orbit coupling that lies at the origin of substantial bonddependent anisotropic Kitaev exchange terms, they are tamed by the Heisenberg exchange term in this system which leads to magnetic long-range order. More specifically, the introduction of the heavy Ag atom in ALIO in place of $\mathrm{Li}$ in $\alpha-\mathrm{Li}_{2} \mathrm{IrO}_{3}$ or the light $\mathrm{H}$ atom in HLIO strongly affects the local structure and enhances the interlayer Ag- $d$ and O- $p$ hybridization along linear $\mathrm{O}-\mathrm{Ag}-\mathrm{O}$ bonds. The increased bond angle originating due to $\mathrm{Ag}-\mathrm{O}$ electronic repulsion essentially contributes to the nearest-neighbor Kitaev exchange. The nearest-neighbor Kitaev and Heisenberg exchanges are found to be ferromagnetic and antiferromagnetic, respectively. Although a comparison of the energy among various magnetic configurations within the unit cell finds the Néel state to be the lowest in energy, the stripy ordered state is not very far in energy (Fig. 9). Both $J$ and $K$ parameters strongly depend on the local geometry, an interesting aspect that can be explored in future studies by the tuning of bond length and bond angles driven by pressure or the application of a magnetic field. This can open up the possibility of suppressing $J$ to negligible values so that the Kitaev term $K$ dominates the physics of the ground state which might then be a Kitaev spin liquid.

We end with the most unusual finding from our study: the continued presence of a high muon relaxation rate $(\sim 5 \mathrm{MHz})$ deep into the ordered state as exemplified by Fig. 4. Our observations go down to temperatures as low as $52 \mathrm{mK}$, which is $1 / 200$ th of the ordering temperature as highlighted earlier in the Introduction and the text surrounding Fig. 4. This points to considerable dynamics of the ordered moments even in the ground state. The $\alpha-\mathrm{RuCl}_{3}$ system also shows a significant muon depolarization rate on the ordered side $[42,43]$; however, as mentioned in Sec. III A, these datasets are limited to about $1 / 5$ th of the ordering temperature. A somewhat similar conclusion of persistent dynamics has been drawn $[44,47]$ based on an analysis of the ${ }^{7} \mathrm{Li}$ NMR $1 / T_{1}$ in the ordered state of ALIO. The natural question is, what is the source of this dynamics? Our data taken together with our computations of the muon stopping site and their dipolar fields are consistent with the coexistence of Néel and stripe ordered domains. We conjecture that the persistent dynamics are due to spatiotemporal fluctuations of these two kinds of ordered domains that are likely driven by quantum effects in the presence of nonnegligible Kitaev terms. This is a well-motivated question for theory; i.e., what is the effect of quantum fluctuations coming from the spin-flip Kitaev terms in the ordered regions of the phase diagram [24]? Ultimately, an explanation for the persistent dynamics, whether driven by Kitaev terms or not, is needed.

\section{ACKNOWLEDGMENTS}

We thank Nandini Trivedi and Vikram Tripathi for discussions. We thank MoE and Department of Science and Technology, Government of India for financial support. A.V.M. kindly acknowledges the Alexander von Humboldt Foundation for financial support for the renewed research stay at the University of Augsburg in 2018. N.B. and A.V.M. kindly acknowledge support from the German Research Society (DFG) via TRR80 (Augsburg, Munich). Author group from IIT Bombay acknowledges support of measurement facilities at their institution. Experiments at the ISIS Neutron and Muon Source were supported by a beam-time allocation RB2068009 from the Science and Technology Facilities Council [48]. S.P. acknowledges financial support from IRCC, IIT Bombay (17IRCCSG011) and SERB, DST, India (SRG/2019/001419). I.D. thanks Department of Science and Technology, Technical Research Centre (DST-TRC) and Science and Engineering Research Board (SERB) India (Project No. EMR/2016/005925) for support.
[1] G. Jackeli and G. Khaliullin, Phys. Rev. Lett. 102, 017205 (2009).

[2] J. Chaloupka, G. Jackeli, and G. Khaliullin, Phys. Rev. Lett. 105, 027204 (2010).

[3] J. Chaloupka, G. Jackeli, and G. Khaliullin, Phys. Rev. Lett. 110, 097204 (2013).

[4] Y. S. Hou, J. H. Yang, H. J. Xiang, and X. G. Gong, Phys. Rev. B 98, 094401 (2018).

[5] V. M. Katukuri, S. Nishimoto, V. Yushankhai, A. Stoyanova, H. Kandpal, S. Choi, R. Coldea, I. Rousochatzakis, L. Hozoi, and J. van den Brink, New J. Phys. 16, 013056 (2014).

[6] V. M. Katukuri, R. Yadav, L. Hozoi, S. Nishimoto, and J. van den Brink, Sci. Rep. 6, 29585 (2016).

[7] S. C. Williams, R. D. Johnson, F. Freund, S. Choi, A. Jesche, I. Kimchi, S. Manni, A. Bombardi, P. Manuel, P. Gegenwart, and R. Coldea, Phys. Rev. B 93, 195158 (2016).
[8] S.-H. Do, S.-Y. Park, J. Yoshitake, J. Nasu, Y. Motome, Y. Kwon, D. T. Adroja, D. J. Voneshen, K. Kim, T.-H. Jang, J.-H. Park, K.-Y. Choi, and S. Ji, Nat. Phys. 13, 1079 (2017).

[9] Y. S. Hou, H. J. Xiang, and X. G. Gong, Phys. Rev. B 96, 054410 (2017).

[10] H. Gretarsson, J. P. Clancy, X. Liu, J. P. Hill, E. Bozin, Y. Singh, S. Manni, P. Gegenwart, J. Kim, A. H. Said, D. Casa, T. Gog, M. H. Upton, H.-S. Kim, J. Yu, V. M. Katukuri, L. Hozoi, J. van den Brink, and Y.-J. Kim, Phys. Rev. Lett. 110, 076402 (2013).

[11] S. Manni, S. Choi, I. I. Mazin, R. Coldea, M. Altmeyer, H. O. Jeschke, R. Valentí, and P. Gegenwart, Phys. Rev. B 89, 245113 (2014).

[12] V. Hermann, S. Biswas, J. Ebad-Allah, F. Freund, A. Jesche, A. A. Tsirlin, M. Hanfland, D. Khomskii, P. Gegenwart, R. Valentí, and C. A. Kuntscher, Phys. Rev. B 100, 064105 (2019). 
[13] I. I. Mazin, S. Manni, K. Foyevtsova, H. O. Jeschke, P. Gegenwart, and R. Valentí, Phys. Rev. B 88, 035115 (2013).

[14] S. K. Choi, R. Coldea, A. N. Kolmogorov, T. Lancaster, I. I. Mazin, S. J. Blundell, P. G. Radaelli, Y. Singh, P. Gegenwart, K. R. Choi, S.-W. Cheong, P. J. Baker, C. Stock, and J. Taylor, Phys. Rev. Lett. 108, 127204 (2012).

[15] S. M. Winter, Y. Li, H. O. Jeschke, and R. Valentí, Phys. Rev. B 93, 214431 (2016).

[16] S. Bette, T. Takayama, K. Kitagawa, R. Takano, H. Takagi, and R. E. Dinnebier, Dalton Trans. 46, 15216 (2017).

[17] K. Kitagawa, T. Takayama, Y. Matsumoto, A. Kato, R. Takano, Y. Kishimoto, S. Bette, R. Dinnebier, G. Jackeli, and H. Takagi, Nature (London) 554, 341 (2018).

[18] Y. Li, S. M. Winter, and R. Valentí, Phys. Rev. Lett. 121, 247202 (2018).

[19] R. Yadav, R. Ray, M. S. Eldeeb, S. Nishimoto, L. Hozoi, and J. van den Brink, Phys. Rev. Lett. 121, 197203 (2018).

[20] S. Bette, T. Takayama, V. Duppel, A. Poulain, H. Takagi, and R. E. Dinnebier, Dalton Trans. 48, 9250 (2019).

[21] R. Kumar, T. Dey, P. M. Ette, K. Ramesha, A. Chakraborty, I. Dasgupta, R. Eremina, S. Tóth, A. Shahee, S. Kundu, M. Prinz-Zwick, A. A. Gippius, H. A. Krug von Nidda, N. Büttgen, P. Gegenwart, and A. V. Mahajan, Phys. Rev. B 99, 144429 (2019).

[22] G. Khaliullin, Phys. Rev. Lett. 111, 197201 (2013).

[23] R. Kumar, T. Dey, P. M. Ette, K. Ramesha, A. Chakraborty, I. Dasgupta, J. C. Orain, C. Baines, S. Tóth, A. Shahee, S. Kundu, M. Prinz-Zwick, A. A. Gippius, N. Büttgen, P. Gegenwart, and A. V. Mahajan, Phys. Rev. B 99, 054417 (2019).

[24] J. G. Rau, Eric Kin-Ho Lee, and H.-Y. Kee, Phys. Rev. Lett. 112, 077204 (2014).

[25] H. Nakamura, Y. Kitaoka, T. Iwai, H. Yamada, and K. Asayama, J. Phys.: Condens. Matter 4, 473 (1992).

[26] Y. Kitaoka, H. Nakamura, T. Iwai, K. Asayama, U. Ahlheim, C. Geibel, C. Schank, and F. Steglich, J. Phys. Soc. Jpn. 60, 2122 (1991).

[27] Z. Zihao and P. Lei, Prog. Phys. 40, 143 (2020).

[28] F. Bahrami, W. Lafargue-Dit-Hauret, O. I. Lebedev, R. Movshovich, H.-Y. Yang, D. Broido, X. Rocquefelte, and F. Tafti, Phys. Rev. Lett. 123, 237203 (2019).

[29] F. Bahrami, E. M. Kenney, C. Wang, A. Berlie, O. I. Lebedev, M. J. Graf, and F. Tafti, Phys. Rev. B 103, 094427 (2021).

[30] See Supplemental Material at http://link.aps.org/supplemental/ 10.1103/PhysRevB.104.115106 for details of the analysis of various experimental data as also the details of theoretical calculations, which includes Refs. [49-55].

[31] G. Kresse and J. Hafner, Phys. Rev. B 47, 558 (1993).

[32] G. Kresse and J. Furthmüller, Phys. Rev. B 54, 11169 (1996).

[33] P. E. Blöchl, Phys. Rev. B 50, 17953 (1994).
[34] G. Kresse and D. Joubert, Phys. Rev. B 59, 1758 (1999).

[35] O. K. Andersen and T. Saha-Dasgupta, Phys. Rev. B 62, R16219 (2000).

[36] V. I. Anisimov, J. Zaanen, and O. K. Andersen, Phys. Rev. B 44, 943 (1991).

[37] S. L. Dudarev, G. A. Botton, S. Y. Savrasov, C. J. Humphreys, and A. P. Sutton, Phys. Rev. B 57, 1505 (1998).

[38] R. C. Williams, F. Xiao, I. O. Thomas, S. J. Clark, T. Lancaster, G. A. Cornish, S. J. Blundell, W. Hayes, A. K. Paul, C. Felser, and M. Jansen, J. Phys.: Condens. Matter 28, 076001 (2016).

[39] S. S. Mohd-Tajudin, S. N. A. Ahmad, D. F. Hasan-Baseri, E. Suprayoga, N. Adam, A. F. Rozlan, S. Sulaiman, M. I. Mohamed-Ibrahim, and I. Watanabe, J. Phys.: Conf. Ser. 551, 012052 (2014).

[40] S. Kundu, A. Shahee, A. Chakraborty, K. M. Ranjith, B. Koo, J. Sichelschmidt, M. T. F. Telling, P. K. Biswas, M. Baenitz, I. Dasgupta, S. Pujari, and A. V. Mahajan, Phys. Rev. Lett. 125, 267202 (2020).

[41] S. Kundu, A. Hossain, Pranava Keerthi S., R. Das, M. Baenitz, P. J. Baker, J.-C. Orain, D. C. Joshi, R. Mathieu, P. Mahadevan, S. Pujari, S. Bhattacharjee, A. V. Mahajan, and D. D. Sarma, Phys. Rev. Lett. 125, 117206 (2020).

[42] I. Yamauchi, M. Hiraishi, H. Okabe, S. Takeshita, A. Koda, K. M. Kojima, R. Kadono, and H. Tanaka, Phys. Rev. B 97, 134410 (2018).

[43] F. Lang, P. J. Baker, A. A. Haghighirad, Y. Li, D. Prabhakaran, R. Valentí, and S. J. Blundell, Phys. Rev. B 94, 020407(R) (2016).

[44] J. Wang, W. Yuan, T. Imai, P. M. Singer, F. Bahrami, and F. Tafti, Phys. Rev. B 103, 214405 (2021).

[45] M. J. O’Malley, H. Verweij, and P. M. Woodward, J. Solid State Chem. 181, 1803 (2008).

[46] A. Kumar and V. Tripathi, Phys. Rev. B 102, 100401(R) (2020).

[47] J. Wang, F. Bahrami, W. Yuan, Philip M Singer, Hung-Yu Yang, F. Tafti, and T. Imai, Bull. Am. Phys. Soc. B 39, 00005 (2021).

[48] A. V. Mahajan, K. Yokoyama, V. Siruguri, P. Biswas, and V. Kumar, ISIS Neutron and Muon Source Data Journal (2020).

[49] S. J. Blundell, Contemp. Phys. 40, 175 (1999).

[50] M. Bouvier, P. Lethuillier, and D. Schmitt, Phys. Rev. B 43, 13137 (1991).

[51] E. Holzschuh, A. B. Denison, W. Kündig, P. F. Meier, and B. D. Patterson, Phys. Rev. B 27, 5294 (1983).

[52] A. B. Denison, J. Appl. Phys. 55, 2278 (1984).

[53] S. J. Blundell, Phys. B: Condens. Matter 404, 581 (2009).

[54] O. K. Andersen and O. Jepsen, Phys. Rev. Lett. 53, 2571 (1984).

[55] A. Yaouanc and P. D. de Réotier, Muon Spin Rotation, Relaxation, and Resonance: Applications to Condensed Matter (Oxford University Press, New York, 2011). 